Supporting Information

\title{
Experiments and Simulations Probing Local Domain Bulge and String Assembly of Aligned Nanoplates in a Lamellar Diblock Copolymer
}

\author{
Nadia M. Krook, ‘ Christian Tabedzki, ${ }^{\star}$ Katherine C. Elbert, ${ }^{\S}$ Kevin G. Yager," \\ Christopher B. Murray, *,', Robert A. Riggleman, * $*$ Russell J. Composto*,广 \\ $\dagger^{\dagger}$ Department of Materials Science and Engineering, University of Pennsylvania, \\ Philadelphia, Pennsylvania 19104, United States \\ *Department of Chemical and Biomolecular Engineering, University of Pennsylvania, \\ Philadelphia, Pennsylvania 19104, United States \\ $\S$ Department of Chemistry, University of Pennsylvania, Philadelphia, Pennsylvania, 19104, United States \\ "Center for Functional Nanomaterials, Brookhaven National Lab, Upton, New York 11973, United States
}

\section{Corresponding Authors}

*E-mail: cbmurray@sas.upenn.edu.

*E-mail: rrig@seas.upenn.edu.

*E-mail: composto@seas.upenn.edu. 


\section{Semi-Implicit Euler Update Scheme Details}

For the convergence, a semi-implicit Euler update scheme was used. The updates are performed in Fourier space in order to perform convolutions; transformed variables are represented with a caret ( ${ }^{\wedge}$ ). Let $A$ be the first general component of the diblock, $B$ the second component (corresponding to PS and PMMA, respectively) and $C$ the grafted PEG chains. The update scheme of the compressibility field is

$$
\hat{w}_{+}^{t+1}=\frac{\hat{w}_{+}^{t}-\lambda_{\text {real }}\left(F-L \hat{w}_{+}^{t}\right)}{1+\lambda_{\text {real }} M}
$$

where $\lambda_{\text {real }}$ represents the step size for the real fields and $M, L$, and $F$ are defined as

$$
\begin{gathered}
M=\frac{\rho_{0}}{\kappa}+\hat{h}^{2} \phi_{\mathrm{D}} \rho_{0} N_{\mathrm{D}}\left(\hat{g}_{\mathrm{AA}}+2 \hat{g}_{\mathrm{AB}}+\hat{g}_{\mathrm{BB}}\right)+\phi_{\mathrm{C}} N_{\mathrm{C}} \hat{g}_{\mathrm{C}} \rho_{0} \hat{h}^{2} \\
L=\hat{h}^{2} \phi_{\mathrm{D}} \rho_{0} N_{\mathrm{D}}\left(\hat{g}_{\mathrm{AA}}+2 \hat{g}_{\mathrm{AB}}+\hat{g}_{\mathrm{BB}}\right)+\phi_{\mathrm{C}} N_{\mathrm{C}} \hat{g}_{\mathrm{C}} \rho_{0} \hat{h}^{2} \\
F=-i \rho_{0} \delta(\mathbf{k})+i\left(\hat{\rho}_{\mathrm{A}, \mathrm{c}} \hat{h}+\hat{\rho}_{\mathrm{B}, \mathrm{c}} \hat{h}+\hat{\rho}_{\mathrm{C}, \mathrm{c}} \hat{h}\right)
\end{gathered}
$$

where $\hat{h}$ is the Fourier transform of

$$
h=\left(\frac{1}{2 \pi a^{2}}\right)^{\frac{d}{2}} \exp \left(-\frac{|\boldsymbol{r}|^{2}}{2 a^{2}}\right)
$$

and is used to "smear" the microscopic densities of the nanoparticles (NPs) from point densities; $d$ represents the system dimensionality. In the update scheme, $\hat{g}$ is the Fourier transform of the Debye function, ${ }^{1} N_{\mathrm{D}}$ is the degree of polymerization of the block copolymer (BCP), $N_{\mathrm{C}}$ is the degree of polymerization of the grafted polymer brush, $\rho_{\alpha}$ represents the microscopic density of type $\alpha\left\{\rho_{\alpha}=\sum_{i}^{n_{\alpha}} \sum_{j}^{N_{\alpha}} \delta\left(r-r_{i, j}\right)\right\}$, and $n_{\alpha}$ is the number of chains that contain bead type $\alpha . \phi_{\mathrm{D}}$ equals the volume fraction of the BCP chain in the box $\left(\phi_{\mathrm{D}=} \phi_{\mathrm{DA}+} \phi_{\mathrm{DB}}\right)$, and $\phi_{\mathrm{C}}$ equals the volume fraction of the grafted brush. As these updates are in Fourier space, $\delta(\mathbf{k})$ represents the Dirac delta function in Fourier space.

Depending on the sign of $\chi_{\alpha \beta}$, there are two slightly different gradient schemes for $\hat{w}_{\alpha \beta}^{( \pm)}$ due to the two different forms of the Hubbard-Stratonovich equation. For a positive $\chi$ system, the real field is $w_{\alpha \beta}^{(+)}$while $w_{\alpha \beta}^{(-)}$ends up being imaginary. However, when $\chi$ is negative, the real field is $w_{\alpha}^{(-)}$and $w_{\alpha \beta}^{(+)}$is the imaginary field. If the untransformed field is real, then the scheme is relatively simple

$$
\left.\hat{w}_{\alpha \beta}^{( \pm)}\right|_{t+1}=\frac{\left.\hat{w}_{\alpha \beta}^{( \pm)}\right|_{t}+\lambda_{\text {real }}\left(\hat{\rho}_{\alpha, c} \hat{h} \pm \hat{\rho}_{\beta, c} \hat{h}\right)}{1+\lambda_{\text {real }}{\underline{\rho_{\alpha \beta}}}_{\chi_{\alpha \beta}}}
$$


If the field is imaginary, the update becomes mildly more complicated

$$
\left.\hat{w}_{\alpha \beta}^{( \pm)}\right|_{t+1}=\frac{\left.\hat{w}_{\alpha \bar{\beta}}^{( \pm)}\right|_{t}-\lambda_{\text {imag }}\left(F-\left.L \hat{w}_{\alpha \bar{\beta}}^{( \pm)}\right|_{t}\right)}{1+\lambda_{\text {imag }} M}
$$

where $\lambda_{\text {imag }}$ is the step size for the imaginary field, and where

$$
\begin{gathered}
F=+i\left(\hat{\rho}_{\alpha, c} \hat{h} \pm \hat{\rho}_{\beta, c} \hat{h}\right) \\
M=\frac{2 \rho_{0}}{\chi_{\alpha \beta}}+L
\end{gathered}
$$

The form of $L$ depends on whether $\alpha$ and $\beta$ are the same chain or not. If they are on the same chain the form is

$$
L=\hat{h}^{2} \phi_{\mathrm{D}} \rho_{0} N_{\mathrm{D}}\left(\hat{g}_{\mathrm{AA}}+\hat{g}_{\mathrm{AB}} \pm\left(\hat{g}_{\mathrm{AB}}+\hat{g}_{\mathrm{AB}}\right)\right)
$$

If $\alpha$ and $\beta$ reside on separate chains, then the form is

$$
L=\hat{h}^{2} \phi_{\mathrm{D}} N_{\mathrm{D}}\left(\hat{g}_{\alpha \alpha}\right) \pm \hat{h}^{2} \phi_{\mathrm{G}} \rho_{0} N_{\mathrm{G}} \hat{g}_{\mathrm{G}}
$$

where $g_{\mathrm{G}}$ is the Debye function applied for the grafted homopolymer, $g_{\mathrm{AB}}$ is the Debye function applied for the whole $\mathrm{BCP}, g_{\mathrm{AA}}$ is the Debye function applied for the $A$ component of the $\mathrm{BCP}$, and $g_{\mathrm{BB}}$ is the Debye function applied for the $B$ component of the BCP. 


\section{Experimental Section}

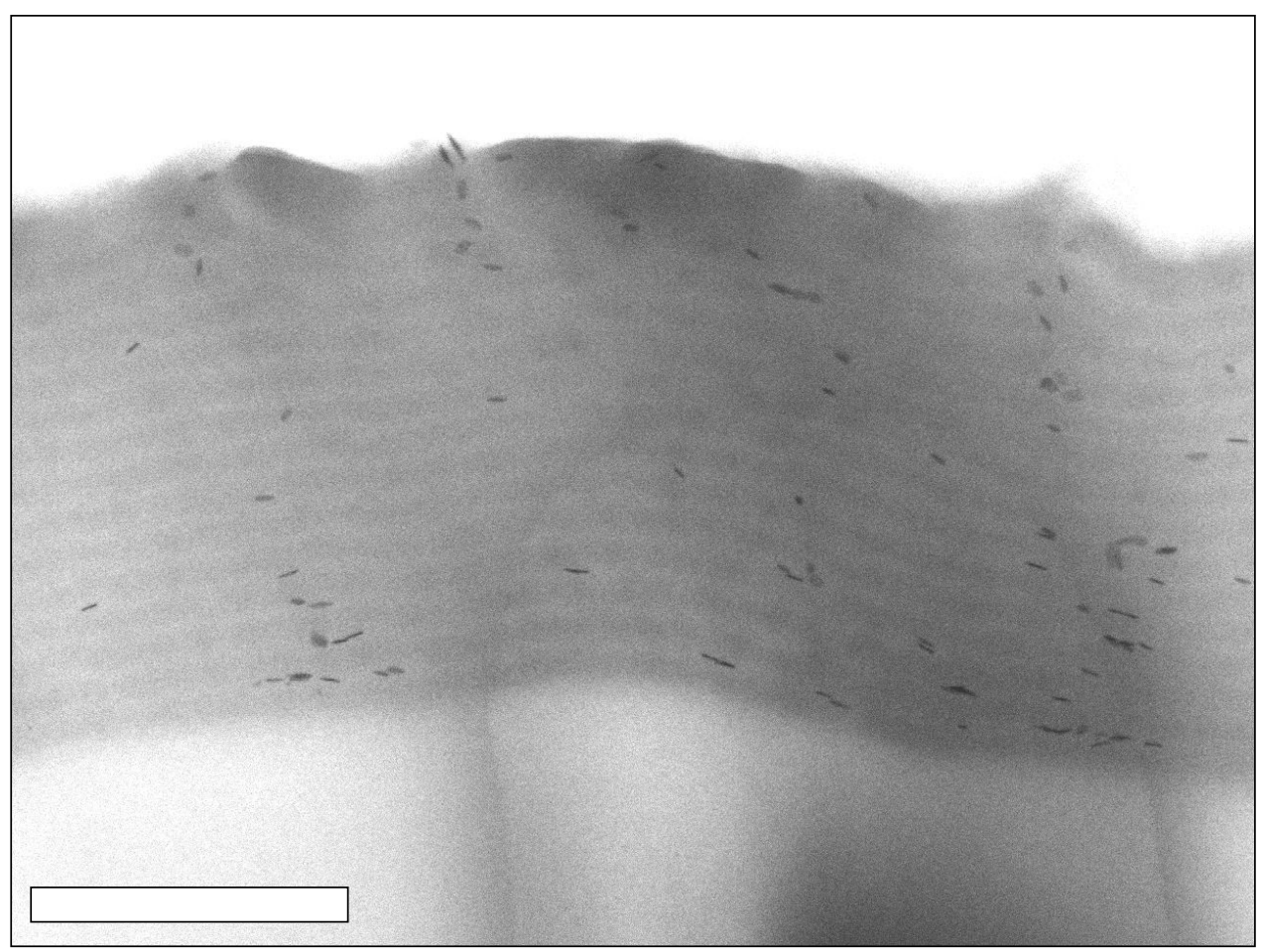

Figure S1. Representative cross-sectional scanning transmission electron microscopy (STEM, JEOL-7500F HRSEM, $30 \mathrm{kV})$ image of annealed $\left(190^{\circ} \mathrm{C}, 48 \mathrm{~h}\right)$ and ultramicrotome lamellar-forming $M_{\mathrm{n}}=38 \mathrm{k}-b-36.8 \mathrm{k} \mathrm{g} / \mathrm{mol}$ PS- $b$-PMMA films (thickness $\sim 550 \mathrm{~nm}$ ) with $\phi=0.0083 M_{\mathrm{n}}=5 \mathrm{~kg} / \mathrm{mol}$ PEG- $\mathrm{PO}_{3} \mathrm{H}_{2}$ functionalized $\mathrm{GdF}_{3}: \mathrm{Yb} / \mathrm{Er}(20 / 2 \mathrm{~mol} \%)$ nanoplates. The light domain is PMMA and the dark domain is PS. The scale bar is $300 \mathrm{~nm}$. 


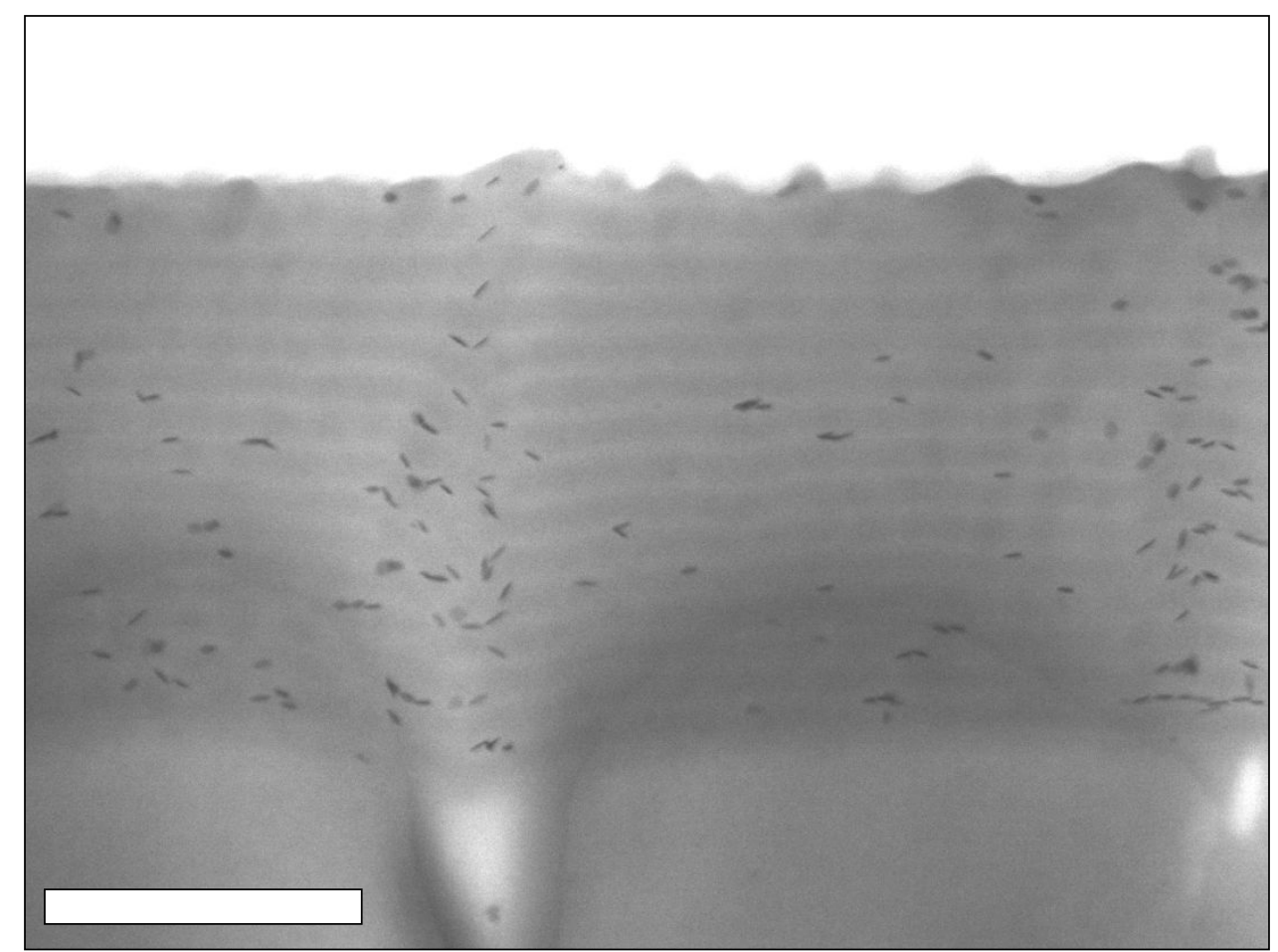

Figure S2. Representative cross-sectional scanning transmission electron microscopy (STEM, JEOL-7500F HRSEM, $30 \mathrm{kV})$ image of annealed $\left(190^{\circ} \mathrm{C}, 48 \mathrm{~h}\right)$ and ultramicrotome lamellar-forming $M_{\mathrm{n}}=38 \mathrm{k}-b-36.8 \mathrm{k} \mathrm{g} / \mathrm{mol}$ PS- $b$-PMMA films (thickness $\sim 540 \mathrm{~nm}$ ) with $\phi=0.017 M_{\mathrm{n}}=5 \mathrm{~kg} / \mathrm{mol} \mathrm{PEG} \mathrm{PO}_{3} \mathrm{H}_{2}$ functionalized $\mathrm{GdF}_{3}: \mathrm{Yb} / \mathrm{Er}(20 / 2 \mathrm{~mol} \%)$ nanoplates. The light domain is PMMA and the dark domain is PS. The scale bar is $300 \mathrm{~nm}$. 

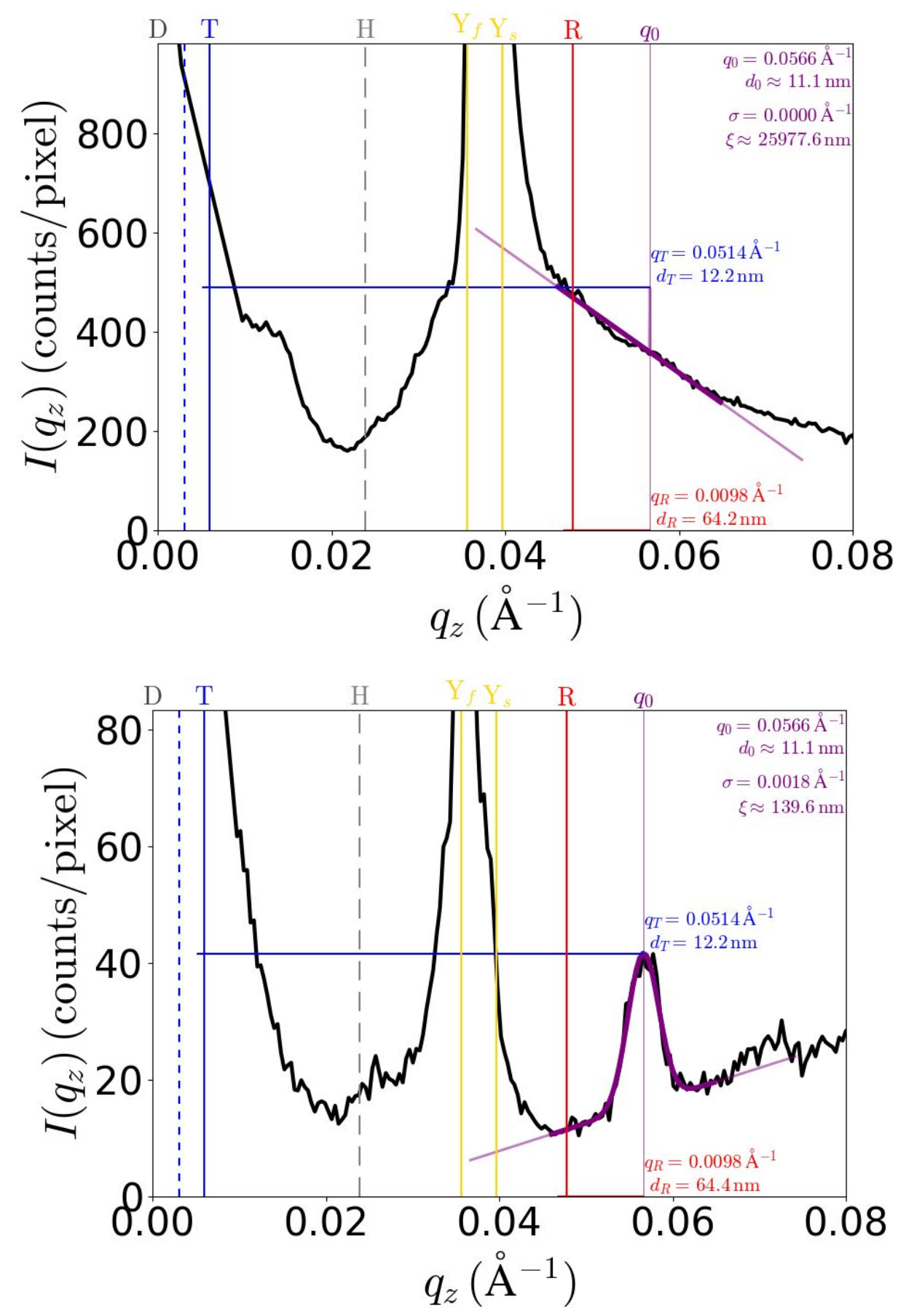

Figure S3. Examples of typical peak analysis to determine the out-of-plane $\left(q_{\mathrm{z}}\right.$ direction) repeat-spacing. The extracted scattering intensity (black line) is fit to a Gaussian peak (purple), from which we extract a nominal peak position $\left(q_{0}\right)$. Based on the known grazing-incident angle and material critical angle, we compute refraction corrections. Various features along $q_{\mathrm{z}}$ are noted: the position of the direct beam $(D)$, the transmitted/refracted beam $(T)$, the sample horizon $(H)$, the Yoneda of the film $\left(Y_{\mathrm{f}}\right)$ and substrate $\left(Y_{\mathrm{s}}\right)$, and the reflected beam $(R)$. From the nominal peak position, a corrected peak position can be computed by applying a refraction correction; the correction assuming the peak comes from the transmitted $\left(q_{\mathrm{T}}\right)$ or reflected beam $\left(q_{\mathrm{R}}\right)$ is different. In the present analysis we confirmed that the peaks of interest arose from the direct beam, and thus convert this corrected peak position $\left(q_{\mathrm{T}}\right)$ into real-space distance $\left(d_{\mathrm{T}}=\right.$ $\left.2 \pi / q_{\mathrm{T}}\right)$. The top analysis is representative scattering data for a sample with disordered lamellae $(\phi=0.050 \mathrm{GdF}: \mathrm{Yb} / \mathrm{Er}$ nanoplates) where the lamellar scattering peak was not observed and the bottom analysis shows a representative peak fit for a sample with ordered lamellae ( $\phi=0 \mathrm{GdF}_{3}: \mathrm{Yb} / \mathrm{Er}$ nanoplates $)$. 


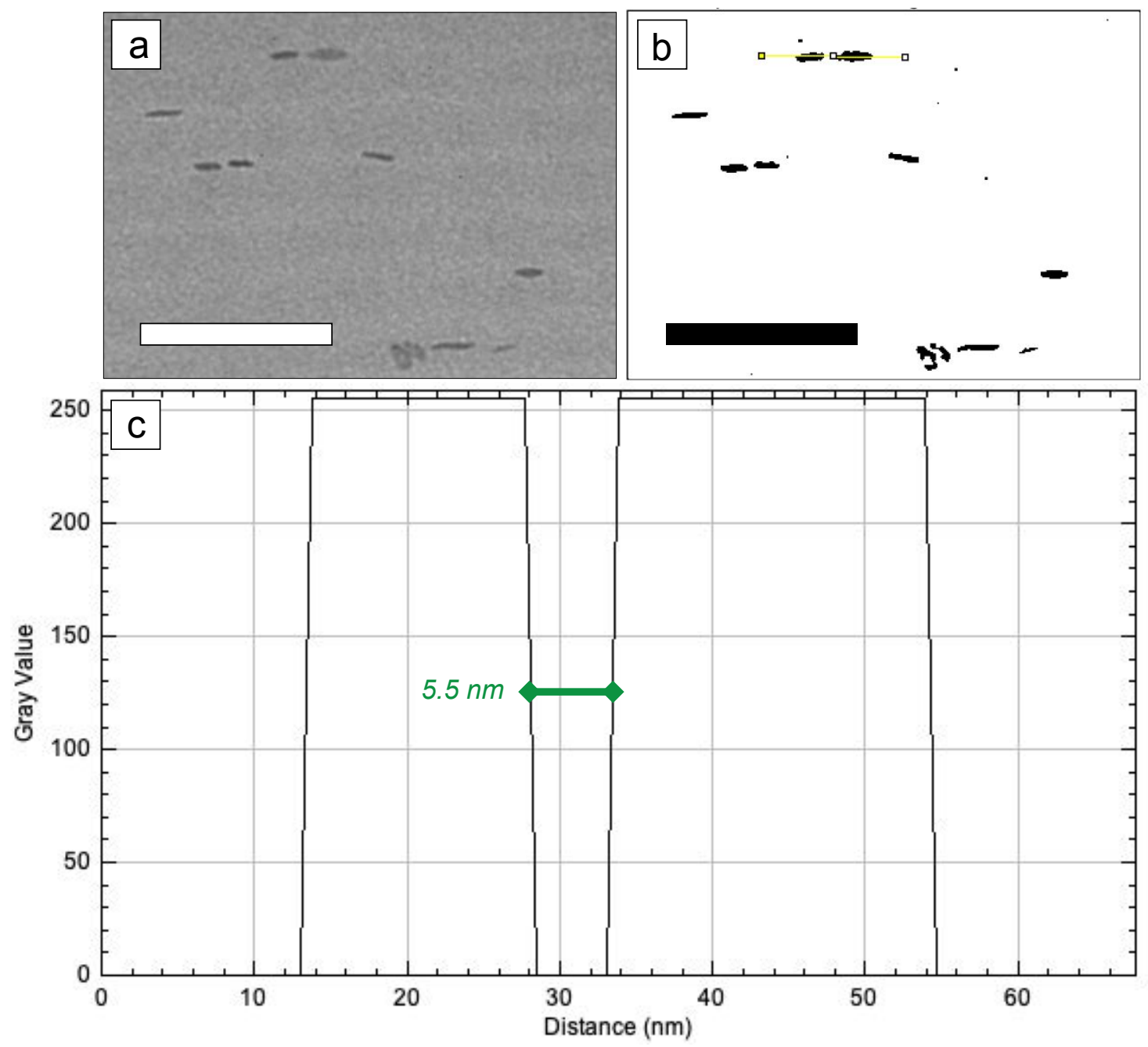

Figure S4. Characteristic ImageJ (v 1.52a) analysis to determine edge-to-edge separation of aligned nanoplates within assembled strings in PMMA domains of $M_{\mathrm{n}}=38 \mathrm{k}-b-36.8 \mathrm{k} \mathrm{g} / \mathrm{mol}$ PS- $b$-PMMA lamellae. (a) TEM image featuring a region of the BCP nanocomposite film with $\phi=0.017$ NPs that demonstrates nanoplates bridging together. (b) The greyscale TEM image from (a) adjusted to a binary image $(\mathrm{NP}=255$ and background $=0$ ) by manual thresholding which also includes a straight line selection drawn along one of the nanoplate strings. (c) The line scan from (b) converted to an intensity as a function of distance profile to measure nanoplate edge separation at half the maximum intensity value. The scale bars are $100 \mathrm{~nm}$. 
Table S1. 300 measurements in nm of the edge-to-edge distance between NPs bridging together in strings in a film of $\phi=0.017 M_{\mathrm{n}}=5 \mathrm{~kg} / \mathrm{mol}$ PEG- $\mathrm{PO}_{3} \mathrm{H}_{2}$ functionalized $\mathrm{GdF}_{3}: \mathrm{Yb} / \mathrm{Er}(20 / 2 \mathrm{~mol} \%)$ nanoplates aligned in ordered $M_{\mathrm{n}}=$ $38 \mathrm{k}-b-36.8 \mathrm{k}$ g/mol PS- $b$-PMMA lamellae, determined via the process illustrated in Figure S4.

\begin{tabular}{|c|c|c|c|c|c|c|c|c|c|}
\hline 5.25 & 7.80 & 4.53 & 9.61 & 5.14 & 6.02 & 5.09 & 5.25 & 6.02 & 5.81 \\
\hline 5.42 & 4.72 & 5.51 & 7.61 & 6.86 & 6.12 & 6.36 & 7.57 & 6.31 & 5.33 \\
\hline 4.65 & 8.96 & 7.16 & 7.58 & 4.70 & 6.26 & 5.76 & 5.82 & 6.11 & 5.93 \\
\hline 6.00 & 7.64 & 8.35 & 8.49 & 6.78 & 4.09 & 5.95 & 6.12 & 6.33 & 5.71 \\
\hline 6.34 & 7.10 & 7.09 & 10.08 & 10.45 & 6.26 & 6.14 & 5.81 & 6.45 & 5.78 \\
\hline 6.79 & 5.38 & 7.19 & 5.40 & 10.92 & 4.44 & 5.13 & 8.50 & 6.17 & 5.97 \\
\hline 7.67 & 6.11 & 6.68 & 8.63 & 6.52 & 7.04 & 4.91 & 5.54 & 6.13 & 7.60 \\
\hline 6.67 & 7.03 & 3.97 & 6.78 & 5.83 & 6.07 & 6.95 & 6.27 & 6.22 & 6.46 \\
\hline 5.40 & 8.92 & 4.50 & 6.27 & 8.94 & 6.75 & 4.26 & 5.21 & 6.86 & 6.29 \\
\hline 7.22 & 10.10 & 7.89 & 6.19 & 6.46 & 6.36 & 6.17 & 5.98 & 6.23 & 4.63 \\
\hline 7.29 & 10.38 & 3.89 & 6.71 & 8.31 & 5.43 & 6.54 & 5.45 & 6.15 & 6.41 \\
\hline 8.06 & 8.12 & 3.37 & 6.45 & 8.63 & 7.24 & 6.13 & 5.71 & 8.45 & 5.79 \\
\hline 5.53 & 3.89 & 8.36 & 6.58 & 6.04 & 8.32 & 4.58 & 6.07 & 8.24 & 5.18 \\
\hline 7.10 & 4.56 & 9.15 & 7.57 & 5.82 & 7.52 & 6.13 & 7.66 & 7.06 & 6.33 \\
\hline 7.77 & 4.88 & 7.60 & 4.93 & 4.53 & 6.49 & 6.02 & 5.46 & 5.46 & 6.55 \\
\hline 7.64 & 3.86 & 5.22 & 9.50 & 5.26 & 6.03 & 6.35 & 5.80 & 6.44 & 5.15 \\
\hline 8.94 & 9.68 & 4.50 & 6.57 & 6.27 & 6.94 & 8.54 & 6.91 & 5.53 & 6.38 \\
\hline 6.45 & 6.48 & 11.60 & 7.63 & 7.48 & 9.11 & 5.58 & 5.92 & 6.30 & 4.98 \\
\hline 9.04 & 5.11 & 6.43 & 6.24 & 8.93 & 6.55 & 5.62 & 6.05 & 4.23 & 4.17 \\
\hline 4.50 & 6.99 & 5.18 & 5.18 & 6.50 & 6.70 & 5.28 & 7.67 & 6.08 & 7.34 \\
\hline 3.89 & 10.79 & 5.83 & 5.37 & 5.91 & 7.49 & 5.23 & 6.32 & 6.75 & 4.23 \\
\hline 6.97 & 6.55 & 6.10 & 5.41 & 7.05 & 6.04 & 4.71 & 9.07 & 7.16 & 5.55 \\
\hline 3.93 & 6.05 & 8.16 & 8.13 & 6.10 & 6.08 & 3.93 & 4.70 & 7.72 & 6.74 \\
\hline 5.57 & 6.55 & 7.09 & 6.57 & 6.39 & 6.26 & 6.60 & 5.77 & 8.16 & 7.45 \\
\hline 5.69 & 3.85 & 8.27 & 8.20 & 6.84 & 8.12 & 5.18 & 6.04 & 6.08 & 5.02 \\
\hline 9.79 & 2.75 & 3.85 & 7.48 & 6.39 & 5.04 & 4.99 & 4.02 & 5.98 & 5.50 \\
\hline 8.49 & 5.03 & 7.26 & 5.24 & 6.48 & 8.00 & 6.59 & 6.61 & 6.31 & 6.01 \\
\hline 8.04 & 5.23 & 3.98 & 5.78 & 7.94 & 6.51 & 8.58 & 6.03 & 6.45 & 6.38 \\
\hline 4.53 & 6.47 & 7.85 & 7.82 & 7.13 & 6.00 & 5.76 & 6.01 & 5.67 & 6.75 \\
\hline 5.30 & 3.65 & 8.39 & 4.01 & 6.62 & 5.42 & 4.55 & 5.42 & 6.90 & 6.18 \\
\hline
\end{tabular}




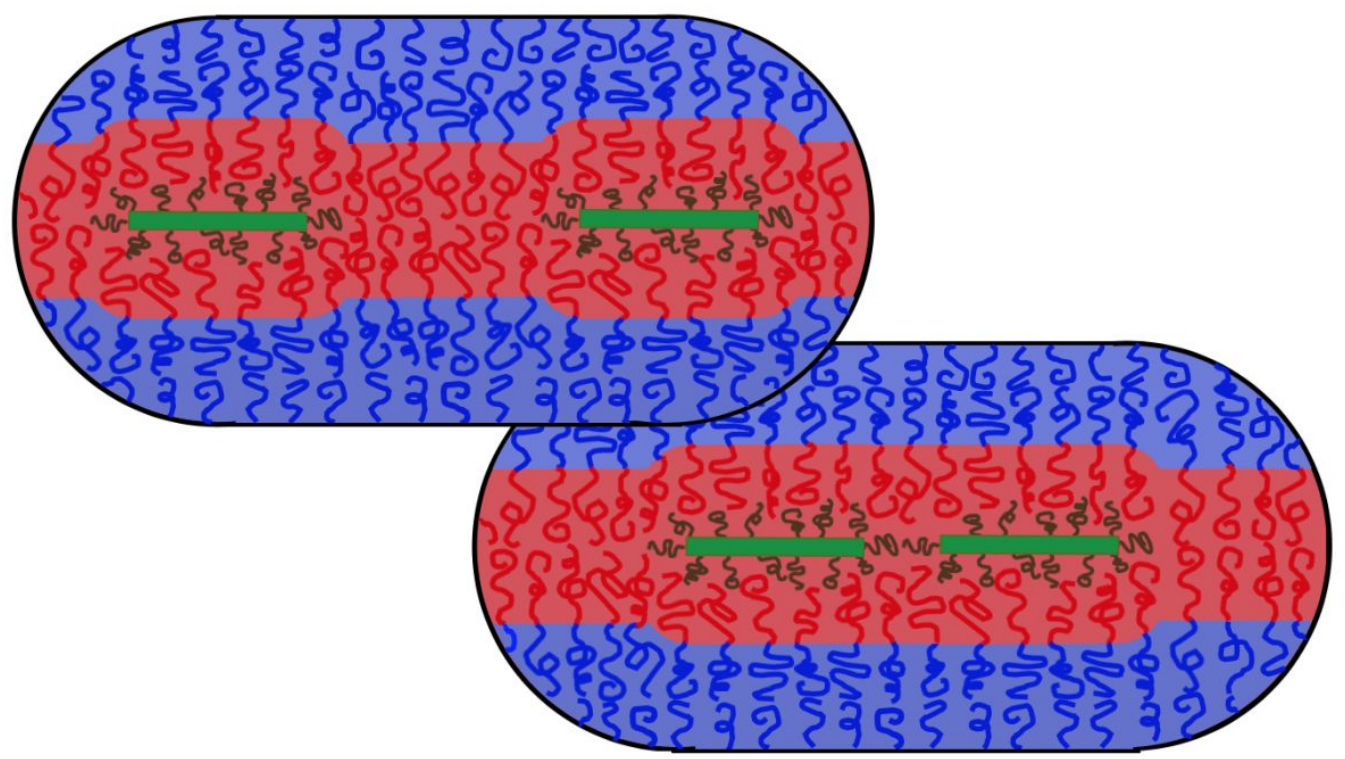

Figure S5. Illustration of two nanoplates stringing together to reduce the total interfacial area between the PMMA (red) and PS (blue) domains by merging the local bulges created around the NPs. 

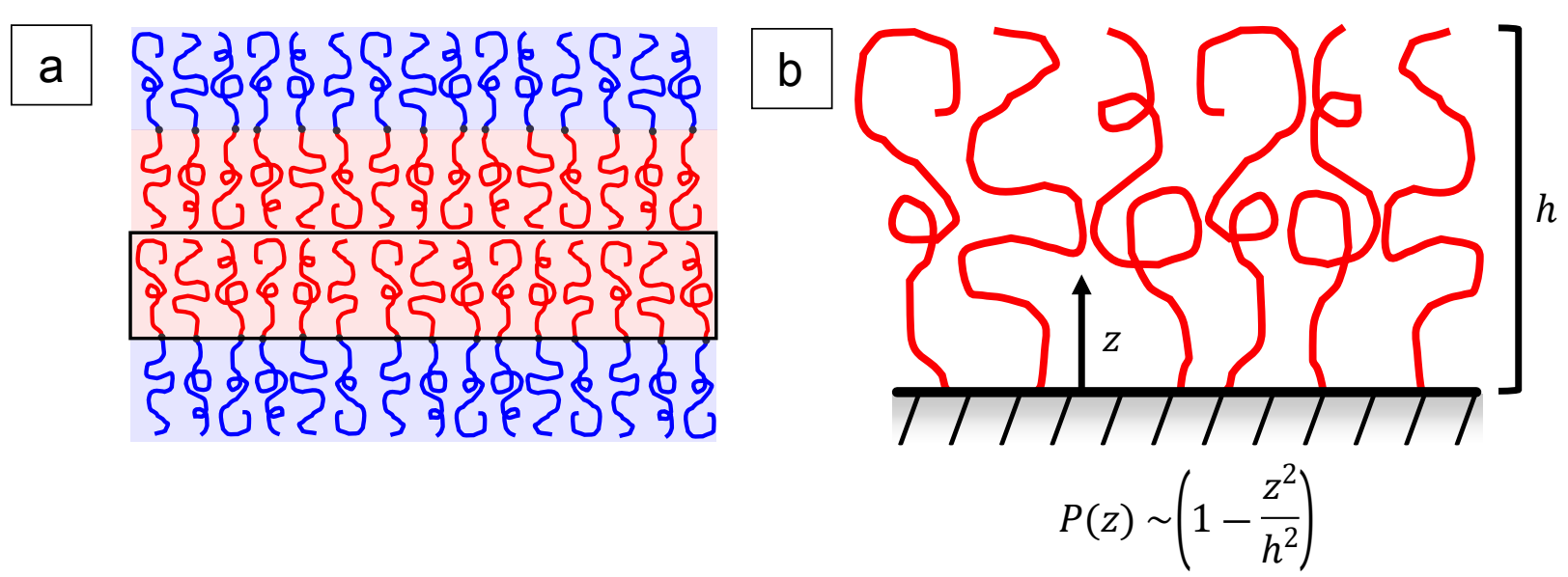

Pryamitsyn, V. and Ganesan, V. Macromolecules 2006, 39, 8499-8510.
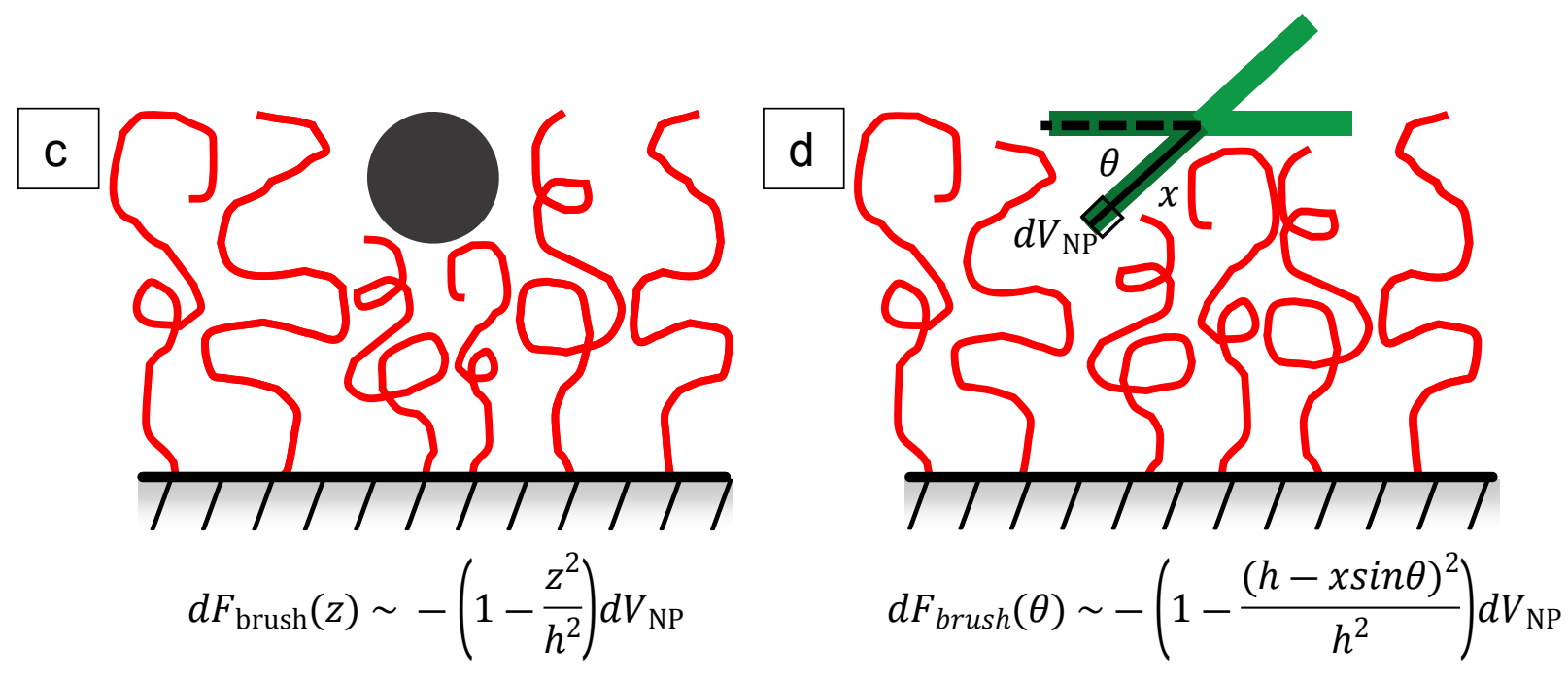

Figure S6. Illustration of (a) the region of an A-B lamellar-forming BCP that is analogized by Pryamitsyn and Ganesan as (b) polymer brushes grafted onto a flat substrate where the pressure field $\left[P(z)\right.$ in $k_{\mathrm{B}} T$ units] on a polymer brush with height $h$ at a position $z$ from the substrate is described as $P(z) \sim\left(1-\frac{z^{2}}{h^{2}}\right)$ and can be modified to $d F_{\text {brush }}(z) \sim-\left(1-\frac{z^{2}}{h^{2}}\right) d V_{\mathrm{NP}}$ when a spherical NP is embedded into the brush layer as in (c). ${ }^{2}$ The model for (c) can then be generalized to $d F_{b r u s h}(\theta) \sim-\left(1-\frac{(h-x \sin \theta)^{2}}{h^{2}}\right) d V_{\mathrm{NP}}$ for (d) when a nanoplate with a differential volume element at distance $x$ along a nanoplate is rotated $\theta$. 


\section{References}

1. Fredrickson, G. H. The Equilibrium Theory of Inhomogeneous Polymers; Oxford University Press: 2006.

2. Pryamitsyn, V.; Ganesan, V. Strong Segregation Theory of Block Copolymer-Nanoparticle Composites. Macromolecules 2006, 39, 8499-8510. 